\title{
CheckMate 067-frontline nivolumab improves PFS alone or in combination with ipilimumab
}

l:

$\mathrm{n}$ the era of immunotherapy

breakthroughs, the results of the

CheckMate 067 trial crown PD-1 inhibitors as the new standard of care for patients with metastatic melanoma, a disease with a very dismal prognosis.

The field of melanoma has witnessed incredible progress in the past 5 years, owing to the approval of agents targeting $B R A F$ mutated disease and the immune checkpoint regulators cytotoxic T-lymphocyte-associated antigen 4 (CTLA-4) and programmed death 1 (PD-1). Both CTLA-4 and PD-1-blocking antibodies-ipilimumab and nivolumab, respectively-have demonstrated improved survival in patients with melanoma; preclinical and early stage studies also indicate a synergistic effect of this combination. Building on these findings, the group of researchers led by Jedd Wolchok conducted a double-blind phase III trial, randomizing 945 previously untreated patients with unresectable stage III or IV melanoma to receive nivolumab alone, ipilimumab alone or a combination of nivolumab plus ipilimumab. Treatments were administered until disease progression or unacceptable toxicity; co-primary end points were progression-free survival (PFS) and overall survival.

While overall survival data are not mature yet, the investigators have reported a median PFS of 11.5 months for patients in the combination arm versus 6.9 months in the single-agent nivolumab arm and 2.9 months in the ipilimumab alone group. Furthermore, objective response rates (ORR) were $57.6 \%$ and $43.7 \%$ for the nivolumab combination and single-agent arms, respectively, and $19 \%$ for the ipilimumab arm. Thus, "nivolumab alone and nivolumab with ipilimumab significantly improved PFS compared to ipilimumab alone in patients with previously untreated melanoma," emphasizes Wolchok. He continues, "the combination resulted

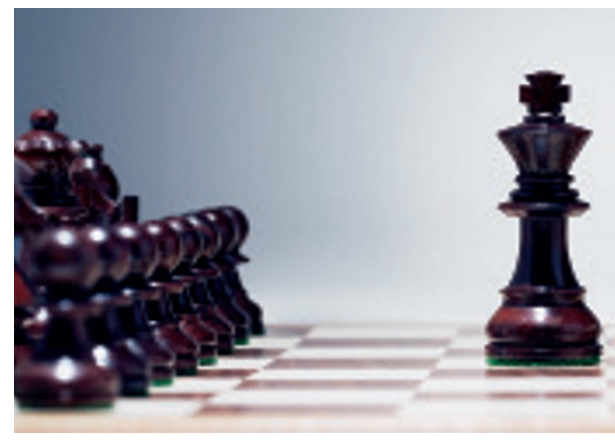

in a numerically higher PFS and a higher ORR versus nivolumab alone." However, the trial was not statistically powered for this comparison.

Subgroup analysis has revealed that patients with tumours positive $(\geq 5 \%)$ for PD-1 ligand, PD-L1, expression have a PFS of 14 months in both nivolumab arms, and 3.9 months with ipilimumab alone. However, PD-L1-negative patients seem to benefit more from the combination approach with a PFS of 11.2 months versus 5.3 months and 2.8 months in the singleagent nivolumab and ipilimumab arms, respectively. The response rates are generally higher for patients who have PD-L1 positive melanoma, with the highest response rate of $72 \%$ in the combination arm, versus $57.5 \%$ in the nivolumab alone arm and $21.3 \%$ in the ipilimumab group.

It is worth mentioning that the CTLA-4 and PD-1 combination caused more toxicity than either monotherapy, with grade 3-4 events in $55 \%$ of patients compared with $16.3 \%$ or $27.3 \%$ of patients treated with nivolumab or ipilimumab monotherapy, respectively. The safety profile of the combination therapy was consistent with data previously reported for the single agents. Importantly, adverse events were manageable and there were no drug-related deaths in the combination group. Wolchok and colleagues have also reported that treatment-related discontinuations were $36.4 \%, 7.7 \%$, and $14.8 \%$ in the combination and

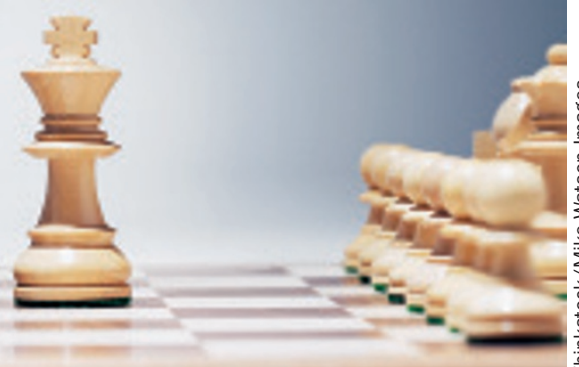

single-agent nivolumab and ipilimumab arms, respectively. Of note, "there was $68 \%$ response rate among the group of patients who discontinued the combination regimen, with half of those responses occurring after the patient stopped receiving treatment," comments Wolchok.

In conclusion, longer PFS and high ORR can be achieved with nivolumab alone or in combination with ipilimumab compared with ipilimumab monotherapy. While data on overall survival are eagerly waited, these initial findings suggest that the combination therapy can be safely used in the clinic. The question remains about those patients that would be best to receive the combination regimen or the PD-1 monotherapy. In this context, perhaps, the use of PD-L1 as a biomarker can inform on the clinical decision-making process.

Ipilimumab is approved in the frontline melanoma setting, whereas nivolumab is approved for metastatic melanoma that progressed following treatment with ipilimumab or a BRAF inhibitor. The CheckMate 067 data strongly support the use of nivolumab as frontline therapy and confirm that the nivolumab-ipilimumab combination is an incredibly promising approach for the treatment of melanoma.

Alessia Errico

Original article Larkin, J. et al. Combined nivolumab and ipilimumab or monotherapy in untreated melanoma. N. Engl. J. Med. doi:10.1056/NEJMoa1504030 